\title{
Collision tumor of small cell carcinoma and squamous cell carcinoma of the maxillary sinus: Case report
}

\author{
IRFAN SUGIANTO $^{1}$, YOSHINOBU YANAGI ${ }^{2}$, MIKI HISATOMI ${ }^{3}$, SHUNSUKE OKADA $^{3}$, \\ YOHEI TAKESHITA ${ }^{4}$, BABATUNDE OLAMIDE BAMGBOSE ${ }^{4}$ and JUNICHI ASAUMI ${ }^{3,4}$ \\ ${ }^{1}$ Department of Oral Radiology Faculty of Dentistry Hasanuddin University, \\ Makassar, Sulawesi 90245, Indonesia; ${ }^{2}$ Department of Dental Informatics, \\ Okayama University Graduate School of Medicine, Dentistry and Pharmaceutical Science; \\ ${ }^{3}$ Department of Oral Diagnosis and Dentomaxillofacial Radiology, Okayama University Hospital; \\ ${ }^{4}$ Department of Oral and Maxillofacial Radiology Okayama University Graduate School of Medicine, \\ Dentistry and Pharmaceutical Science, Okayama 700-8558, Japan
}

Received July 22, 2021; Accepted January 27, 2022

DOI: $10.3892 / \mathrm{mco} .2022 .2529$

\begin{abstract}
A collision tumor refers to the coexistence of two diagnostically distinct tumors in a common anatomic space. Collision tumors are rare in the oral and maxillofacial region. The present study reported on the case of an 82-year-old female with a collision tumor in the maxillary sinus consisting of small cell carcinoma (SmCC) and squamous cell carcinoma (SCC). Computed tomography (CT) imaging revealed a mass in the right maxillary sinus. The lesion exhibited heterogeneous low signal intensity (SI) on T1-weighted imaging (T1WI), high SI on short T1 inversion recovery and heterogeneous solid enhancement on contrast-enhanced T1WI. The histopathology result of a biopsy specimen confirmed SmCC. After the patient received a course of chemoradiotherapy, follow-up CT revealed a residual tumor. In a second surgery, a remaining tumor and histopathology revealed SCC with no evidence of SmCC. The final diagnosis was a collision tumor made up of SCC and SmCC.
\end{abstract}

\section{Introduction}

A collision tumor is a coexistence of two diagnostically distinct tumors in a common anatomic space (1). Small cell carcinoma (SmCC) is a high-grade tumor derived from neuroendocrine cells. Extrapulmonary SmCC is rare, accounting for $2.5-5 \%$ of all cases of SmCC. The genitourinary and gastrointestinal

Correspondence to: Dr Irfan Sugianto, Department of Oral Radiology Faculty of Dentistry Hasanuddin University, J1. Perintis Kemerdekaan Km 10, Makassar, Sulawesi 90245, Indonesia E-mail: irfansugianto@unhas.ac.id

Key words: multiple primary neoplasms, small cell carcinoma, squamous cell carcinoma, maxillary sinus, magnetic resonance imaging systems are the most common sites (2). In the maxillary sinuses, the most common malignancy is squamous cell carcinoma (SCC), followed by adenocarcinoma $(3,4)$. SmCC is a highly aggressive tumor with high recurrence rates and propensity for distant metastasis, hence its poor prognosis (4).

In the head and neck region, the collision of neuroendocrine tumors is uncommon. Only a small number of cases in the oral region and sinonasal area have been reported $(3,5,6)$. This occurrence has been reported more frequently in the larynx (7). Composite tumor in the sinonasal area mostly comprises adenocarcinoma and neuroendocrine carcinoma (5). Collision tumors made up of SmCC and SCC are rare. The present study reported on a case of SmCC of the maxillary sinus; at the same site, SCC was detected during follow-up. The radiologic findings, including dynamic contrast-enhanced (DCE)-magnetic resonance imaging (MRI), are discussed.

\section{Case report}

An 82-year-old female was referred to Okayama University Hospital (Okayama, Japan) with pain in the upper right gingiva in the proximity of the partial denture with associated cheek swelling. Intra-oral examination revealed a $26-\mathrm{mm}$ compressible swelling of the right cheek and a non-tender mass in the upper right edentulous gingiva extending from the canine region to the molar region. In addition, the Water's projection revealed a radiopaque mass in the right maxillary sinus (Fig. 1A and B). The patient's medical history was significant for hypertension and myocardial infarction.

Axial computed tomography (CT) images displayed a mass in the maxillary sinus with a bony defect of the anterior wall (Fig. 2A). No destruction of the posterior wall, ethmoid sinuses or pterygoid plate was present. On MRI, the mass exhibited heterogeneous enhancement on post-contrast images. MRI examination was performed using a $1.5 \mathrm{~T}$ device (Magnetom Vision ${ }^{\circledR}$; Siemens AG) with a head and neck coil. T1-weighted images (T1WI) were acquired with a spin-echo sequence using repetition time (TR)/echo time (TE) parameters of 450/10 
msec in addition to short $\mathrm{T} 1$ inversion recovery (STIR) images using turbo-spin echo sequence TR/TE/inversion time parameters of 4,500/60/140 msec. In addition, DCE-MRI images were acquired with $3 \mathrm{D}$ fast imaging with a steady-state precession sequence using the following parameters: TR, $5 \mathrm{msec}$; TE, 2 msec; flip angle, $25^{\circ}$; 16 partitions in a 48 slab; section thickness, $3 \mathrm{~mm}$; 250x188-mm rectangular fields of view; and a $256 \times 192$ matrix resulting in a $0.98 \times 0.98-\mathrm{mm}$ pixel size. The first image series was obtained in 14 consecutive scans. Gadolinium-diethylentriamine pentaacetic acid (Magnevist Syringe; Nihon Schering) was administered intravenously for $6 \mathrm{sec}$ at a rate of $\sim 0.2 \mathrm{ml} / \mathrm{kg}$ via manual injection between the first and second scans in the first series. Second- and third-series scans were performed at 440 and $880 \mathrm{sec}$ after the injection. All scans were acquired over $14 \mathrm{sec}$ with a 1-sec interval between each scan.

The mass demonstrated heterogeneous low signal intensity (SI) on T1WI (Fig. 2B), high SI on STIR images (Fig. 2C) and heterogeneous solid enhancement on contrast-enhanced (CE) T1WI (Fig. 2D). Dynamic CE images were analyzed with regions of interest using a workstation (Synapse Vincent $^{\circledR}$; Fujifilm Medical) to obtain contras index curves (CI curves). The CI curves exhibited a rapid increase over $\sim 100 \mathrm{sec}$ and then a further increase without washout of the contrast medium (Fig. 3). The initial radiologic diagnosis was a lymphoproliferative lesion. The DCE-MRI suggested non-SCC with salivary gland tumor and adenocarcinoma differential. On ${ }^{18} \mathrm{~F}$ fluorodeoxyglucose positron-emission tomography (FDG-PET) indicated no other abnormal sites, suggesting that the maxillary sinus mass was a primary lesion with a maximum standardized uptake value (SUVmax) of 24.81 .

Histopathologic examination of a biopsy specimen revealed a large amount of chromatin and non-uniform cells on $\mathrm{H} \& \mathrm{E}$ staining. The maxillary sinus tissue was fixed with formaline and embedded in paraffin $(10 \%)$ at $60^{\circ} \mathrm{C}$ and cut into $2.5-\mu \mathrm{m}$ sections. The sections were incubated with antibodies against CD56 (1:125 dilution; cat. no. 123C3; Roche Diagnostics), Chromogranin A (1:1,000 dilution; cat. no. LH2H10; Roche Diagnostics), Synaptophysin (1:200 dilution; cat. no. SP11; Roche Diagnostics) and Ki-67 (1:500 dilution; cat. no. 30-9; Roche Diagnostics) at $37^{\circ} \mathrm{C}$ for $15 \mathrm{~min}$. Immunostaining indicated that 2 of 3 neuroendocrine tumor markers, CD56 and chromogranin A were expressed, while synaptophysin was negative. However, Ki-67 was positive (Fig. 4A-C). Based on these findings, the case was diagnosed with SmCC.

At seven weeks after the initial visit, the patient underwent concurrent chemoradiotherapy (CCRT). Carboplatin and etoposide were administered intravenously at a dose of 225 and $110 \mathrm{mg} / \mathrm{day}$, respectively. The CCRT procedure was performed for three days with three-week intervals for each cycle with four cycles. The patient also underwent radiotherapy during the chemotherapy course. The total radiotherapy dose was 60 Gy with 30 fractions of 2 Gy given five days per week. The patient had no adverse events associated with CCRT. CT (Fig. 5A) and MRI (Fig. 5B) evaluation indicated a residual tumor on the right of the maxillary sinus. A Denker procedure was performed to excise the residual tumor. Histologic examination of the surgical specimen of residual tumor using H\&E staining
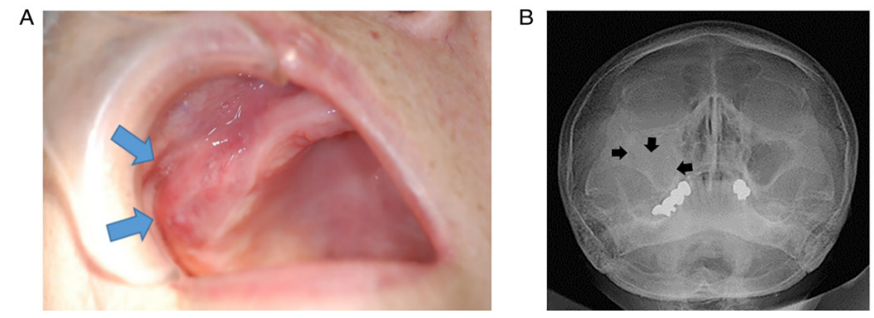

Figure 1. (A) Buccal expansion of the alveolus and obliteration of the sulcus in the upper right canine to the molar region (arrows). (B) Water's view image exhibiting a radiopaque area in the right maxillary sinus (arrows) but no bone destruction in the nasal antrum.
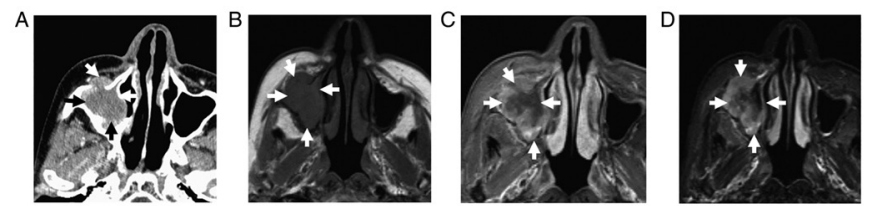

Figure 2. (A) Contrast enhancement computed tomography image indicating heterogeneous enhancement of mass in the right maxillary sinus. (B) Magnetic resonance-T1WI with a slightly homogeneous low signal to intermediate signal intensity. (C) High signal intensity on short T1 inversion recovery. (D) T1WI with contrast indicated strong heterogeneous enhancement. The lesion is indicated with arrows. T1WI, T1-weighted imaging.

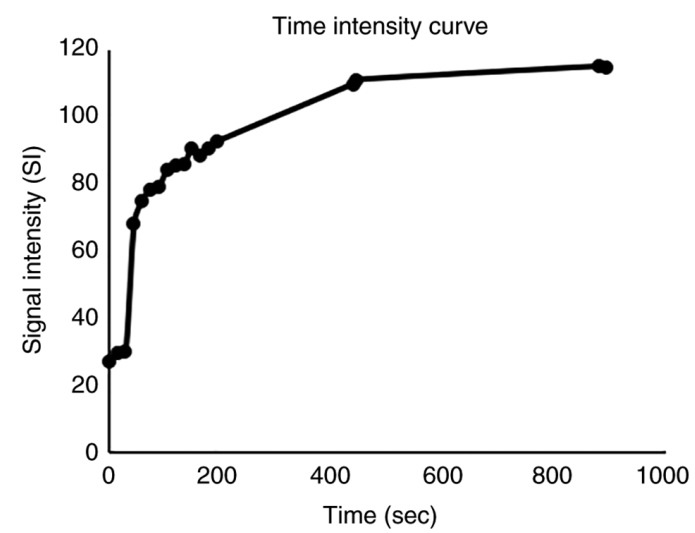

Figure 3. DCE-MRI analysis. The time-intensity curve demonstrates rapid increase and gradual increase without washout. The curve of DCE-MRI analysis revealed non-squamous cell carcinoma and suggested salivary gland tumor and adenocarcinoma. DEC-MRI, dynamic contrast-enhanced magnetic resonance imaging.

(cat. no. H9627; MilliporeSigma) revealed positivity for cytokeratin 7 (CK7; cat. no. M7018; Dako) and CK20 (cat. no. PA0022; Leica Biosystems $\mathrm{GmbH}$ ), consistent with the diagnosis of SCC (Fig. 6). The imunostaining result of neuroendocrine was negative and therefore, SmCC was no longer apparent. The patient underwent chemotherapy using cisplatin. However, treatment was stopped after the first course due to adverse effects of the therapy. Subsequently, the patient regularly underwent follow-ups until six months after the second surgery. ${ }^{18} \mathrm{~F}$ FDG-PET revealed tumor recurrence in the maxillary sinus (SUVmax=6.0) (Fig. 7A) and multiple metastases to organs in the abdomen (Fig. 7B). The patient was transferred to a palliative care facility and died two months after the detection of metastases. 

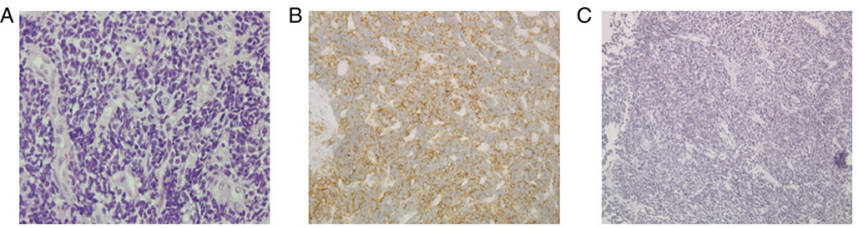

Figure 4. Histopathologic examination revealed a high amount of chromatin and non-uniform cells on H\&E staining. Immunostaining indicated the presence of 2 of 3 neuroendocrine tumor markers, CD56 and Chromogranin. (A) A high N/C ratio and atypical nuclei (H\&E) suggested a high amount of chromatin and non-uniform cells revealed small cell carcinoma. (B) Immunostaining: CD56 (+); (C) Immunostaining: Chromogranin A (+) (magnification, $\mathrm{x} 400$ ).
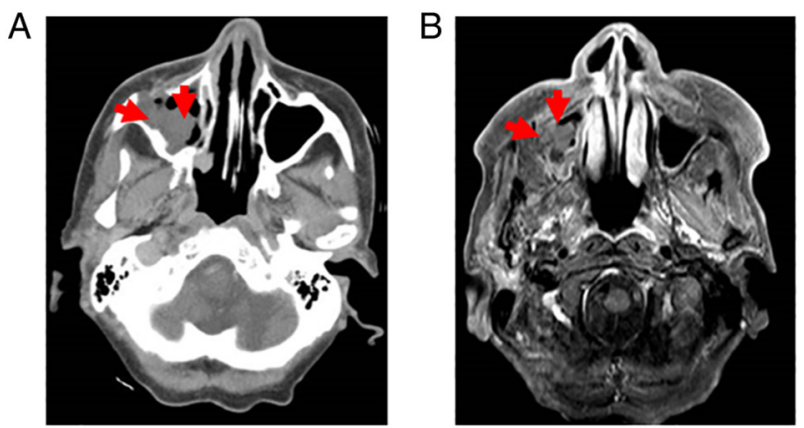

Figure 5. CT and MR imaging after concurrent chemoradiotherapy revealed a remaining tumor. (A) On soft tissue window CT, a mass in the right of the maxillary sinus, was observed, which was a remaining tumor at the exact site of the initial tumor. (B) MR-T1-weighted imaging with contrast displayed heterogeneous enhancement that indicated a mass at the right side of the maxillary sinus (arrows). MR, magnetic resonance; CT, computed tomography.
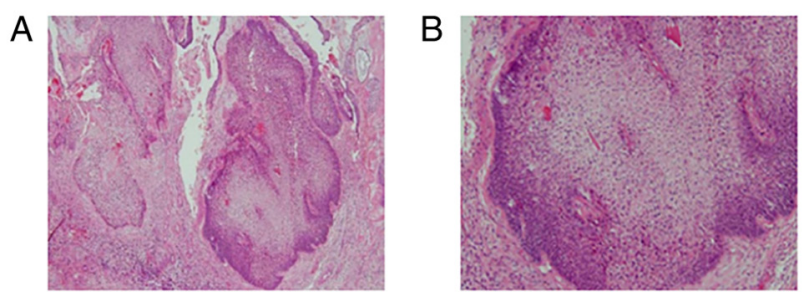

Figure 6. Low-magnification H\&E staining of surgical specimen of residual tumor [H\&E; magnification, (A) x40 and (B) x100] revealed CK7 and CK20 (epithelial tumor marker). CK, cytokeratin.

\section{Discussion}

Collision tumor refers to two malignant tumors coexisting and the components originate from the same area or organ but with different morphologies according to histologic examination. There are various theories related to collision tumors; however, due to low frequency and individuality, controversy still exists regarding the pathogenesis and definition (8). Reports on the synchronous and metachronous coexistence of SmCC and SCC in the same anatomic space in the head and neck are rare. One study proposed two possible explanations for this phenomenon. The first is that the tumors arise from a typical pluripotential stem cell with subsequent divergent differentiation. The second theory is that two separate tumors coincide through two independent molecular processes (3).
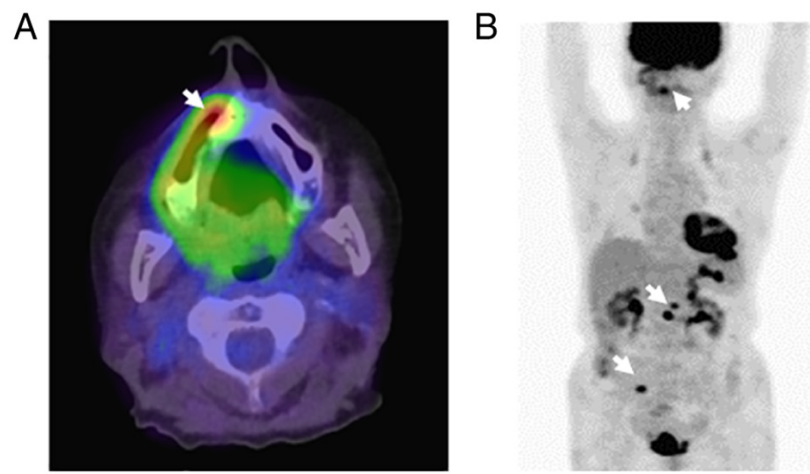

Figure $7 .{ }^{18} \mathrm{~F}$ fluorodeoxyglucose positron-emission tomography indicated the recurrence of the tumor and multiple metastases. (A) Recurrence tumor in the maxillary sinus (maximum standardized uptake value, 6.0). (B) Multiple metastases to organs in the abdomen (arrows).

SmCC of the head and neck is an aggressive tumor type with a propensity to spread locoregionally with simultaneous distant metastases $(3,4)$. Head and neck SmCC is more common in the larynx and rare in the paranasal sinuses (2). The diagnostic markers for neuroendocrine tumors include CD56, chromogranin A and synaptophysin (6,9). Kontogianni et al (10) reported that SmCC was positive for CD56 and negative for chromogranin and synaptophysin.

In the case of the present study, Immunostaining indicateded that CD56 and chromogranin A were positive, while synaptophysin was negative and $\mathrm{Ki}-67$ was positive on initial examination, suggesting the diagnosis of SmCC. Other results on post-CCRT treatment revealed epithelial tumor markers of a high level of squamous cell antigen $(11.3 \mathrm{ng} / \mathrm{ml})$ and a positive result of keratin AE1/AE3 was evident, which was suggestive of SCC, an epithelial tumor. The residual tumor was precisely in the same area of the primary tumor and was a different type of tumor, and it was hypothesized that the occurrence of SCC after CCRT of SmCC may have been due to the initial tumor containing epithelial tumor cells.

On conventional radiographs and $\mathrm{CT}$, the present case exhibited destruction of the anterior wall and tumor progression through an anterior wall. There was no destruction of the posterior wall, ethmoid sinuses or pterygoid plate. Most cases of SmCC in the head and neck extend into adjacent spaces without extensive bone destruction (3). A previous study reported on SmCC of the nasal cavity with extensive and aggressive destruction of bone at the skull base and invasion of the right orbit (8).

The MR findings of SmCC in the paranasal sinuses include moderate SI on T1WI, slightly high SI on T2WI high SI on STIR (11) and mild-to-moderate homogenous enhancement after administration of contrast agent (12). In the present case, the mass demonstrated heterogeneous low-to-moderate SI on T1WI and high SI on STIR images, with contrast-enhanced T1WI revealing intense heterogeneous enhancement. A previous study by our group reported on a case of malignant lymphoma with low SI on T1WI and high SI and slightly high enhancement on T2WI and CE-T1WI, respectively (13). CI curve analysis indicated a rapid increase until $\sim 100 \mathrm{sec}$ and further expansion without washout. This finding is different from other malignancies, such as SCC and malignant 
lymphoma, which rapidly increase and gradually decrease with varying peak times (14).

The limitation of the present study wass that on initial presentation, the entire primary lesion was not examined and the border of each lesion was not defined, but only a biopsy was performed. The histopathological examination only revealed $\mathrm{SmCC}$, although the subsequent result indicated epithelial tumor cells. Histopathologic examination of the complete specimen of is critical to the detection of collision tumor.

\section{Acknowledgements}

Not applicable.

\section{Funding}

No funding was received for this study.

\section{Availability of data and materials}

All data generated or analyzed during this study are included in this published article.

\section{Authors' contributions}

IS: Conceptualization, design, drafting of the manuscript; YY: Acquisition of data and critical revision; $\mathrm{MH}, \mathrm{SO}$, YT and BOB: Conceptualization and critical revision; JA: Acquisition and analysis of the data, critical revision and final approval of the manuscript. All authors read and approved the final manuscript. Data authentication is not applicable.

\section{Ethics approval and consent to participate}

Not applicable.

\section{Patient consent for publication}

The authors have obtained the appropriate consent from the patient's relatives to publish the case report.

\section{Competing interests}

The authors declare that they have no competing interests.

\section{References}

1. Nabili V, Natarajan S, Hirschovitz S, Bhuta S and Abemayor E: Collision tumor of thyroid: Metastatic lung adenocarcinoma plus papillary thyroid carcinoma. Am J Otolaryngol 28: 218-220, 2007.

2. Van der Heijden HF and Heijdra YF: Extrapulmonary small cell carcinoma. South Med J 98: 345-349, 2005.

3. Barham HP, Said S and Ramakrishnan VR: Colliding tumor of the paranasal sinus. Allergy Rhinol (Providence) 4: e13-e16, 2013.

4. Day TA, Beas RA, Schlosser RJ, Woodworth BA, Barredo J, Sharma AK and Gillespie MB: Management of paranasal sinus malignancy. Curr Treat Options Oncol 6: 3-18, 2005.

5. Huang SF, Chuang WY, Cheng SD, Hsin LJ, Lee LY and Kao HK: A colliding maxillary sinus cancer of adenosquamous carcinoma and small cell neuroendocrine carcinoma-a case report with EGFR copy number analysis. World J Surg Oncol 8: 92, 2010.

6. Mochizuki Y, Omura K, Sakamoto K, Nakanishi S, Satoh K, Marukawa E and Yamaguchi A: A case of primary combined neuroendocrine carcinoma with squamous cell carcinoma in the upper gingiva. Oral Surg Oral Med Oral Pathol Oral Radiol Endod 109: e34-e39, 2010.

7. Davies-Husband CR, Montgomery P, Premachandra D and Hellquist H: Primary, combined, atypical carcinoid and squamous cell carcinoma of the larynx: A new variety of composite tumour. J Laryngol Otol 124: 226-229, 2010.

8. Yu Q, Chen YL, Zhou SH, Chen Z, Bao YY, Yang HJ, Yao HT and Ruan LX: Collision carcinoma of squamous cell carcinoma and small cell neuroendocrine carcinoma of the larynx: A case review and review of the literature. World J Clin Cases 7: 242-252, 2019.

9. Hosokawa S, Okamura J, Takizawa Y and Mineta H: Long-term survival of a patient with primary small cell neuroendocrine carcinoma of the maxillary sinus: A case report. J Oral Maxillofac Surg 71: e248-e252, 2013.

10. Kontogianni K, Nicholson AG, Butcher D and Sheppard MN: CD56: A useful tool for the diagnosis of small cell lung carcinomas on biopsies with extensive crush artefact. J Clin Pathol 58: 978-980, 2005.

11. Joyce EA, Kavanagh J, Sheehy N, Beddy P and O'Keeffe SA: Imaging features of extrapulmonary small cell carcinoma. Clin Radiol 68: 953-961, 2013.

12. Zhu Q, Zhu W, Wu J and Zhang H: The CT and MRI observations of small cell neuroendocrine carcinoma in paranasal sinuses. World J Surg Oncol 13: 54, 2015.

13. Matsuzaki H, Hara M, Yanagi Y, Asaumi J, Katase N, Unetsubo T, Hisatomi M, Konouchi H, Takenobu $\mathrm{T}$ and Nagatsuka H: Magnetic resonance imaging (MRI) and dynamic MRI evaluation of extranodal non-Hodgkin lymphoma in oral and maxillofacial regions. Oral Surg Oral Med Oral Pathol Oral Radiol 113: 126-133, 2012.

14. Asaumi J, Yanagi Y, Konouchi H, Hisatomi M, Matsuzaki H and Kishi K: Application of dynamic contrast-enhanced MRI to differentiate malignant lymphoma from squamous cell carcinoma in the head and neck. Oral Oncol 40: 579-584, 2004.

This work is licensed under a Creative Commons Attribution-NonCommercial-NoDerivatives 4.0 International (CC BY-NC-ND 4.0) License. 